\title{
Solid/Gas Concentration Measurements Using Multiple Fan Beam Optical Tomography
}

\author{
R. Abdul Rahim ${ }^{*}$, , L.C. Leong', K.S. Chan ${ }^{1}$, M.H. Fazalul Rahiman² and J.F. Pang ${ }^{2}$ \\ ${ }^{I}$ Process Tomography Research Group (PROTOM), Department of Control \& Instrumentation Engineering, Faculty of \\ Electrical Engineering, Universiti Teknologi Malaysia, 81310 UTM Skudai, Johor, Malaysia \\ ${ }^{2}$ Tomography Imaging Research Group, School of Mechatronic Engineering, Universiti Malaysia Perlis, 02600 Jejawi, \\ Arau, Perlis, Malaysia
}

\begin{abstract}
The application of optical sensors in process tomography involves using non-intrusive sensors to obtain vital information in order to produce images from the dynamic characteristics of a process system. Multiple fan beam projection technique here is defined as allowing more than one emitter to transmit light at the same time using the switch-mode fan beam method. For the thirty-two pairs of sensors used, the 2-projection technique and 4-projection technique are being investigated. Sixteen sets of projections will complete one frame of light emission for the 2-projection technique while eight sets of projection will complete one frame of light emission for the 4-projection technique. The data obtained from optical fibre sensors will then be reconstructed into image using the Linear Back Projection and Iterative Reconstruction algorithms through Visual $\mathrm{C}++$ programming. Concentration measurements and series of error analyses will determine the feasibility of the optical fibre sensors to perform as an instrument in solid flow visualization.
\end{abstract}

\section{INTRODUCTION}

Process tomography is a developing measurement technology. Over the last decade, the concept of tomographic imaging was not restricted to medical field only; it has been successfully developed into a reliable tool for imaging numerous industrial applications. Sensors in the measurement system prove to be the most important part in acquiring the physical signal. The physical signal is then being converted, amplified and fed into the computer to obtain the internal behaviours of the investigated vessel. A conveyor with fast moving flow and rapid changing flow regime require a sensor with very fast response and also a fast data acquisition rate in order to prevent data losses. The optical sensors has proven to have the advantages of fast respond, high performance, low cost and low maintenance compared to electrical capacitance tomography

Parallel projection and fan beam projection are two different types of sensor arrangement methods. For sensor arrangement method using parallel projection method, each transmitter and receiver only corresponds to each other. While for the fan beam projection method, there may be more than one receiver corresponding to a single or multiple light sources. In the switch-mode fan beam method, a multiplexing source is used and all the receivers produce the signals corresponding to each multiplexed source.

Tomographic images are usually derived using certain image reconstruction algorithms. Reconstruction of image is a unique processing problem for an object from a set of

\footnotetext{
*Address correspondence to this author at the Process Tomography Research Group (PROTOM), Department of Control \& Instrumentation Engineering, Faculty of Electrical Engineering, Universiti Teknologi Malaysia, 81310 UTM Skudai, Johor, Malaysia;

E-mail: ruzairiabdulrahim@yahoo.co.uk,ruzairi@fke.utm.my
}

cross-sectional projections data of the object. It is necessary to solve the forward problem first in order to derive the algorithm which provides solutions to the inverse problem. Fig. (1) illustrates the forward problem and inverse problem in optical tomography.

\section{FORWARD PROBLEM}

The forward problem determines the theoretical output of each of the sensors when the sensing area is considered to be two dimensional [1]. The forward problem consists of detailed description of the sample under investigation; and includes its experimental projection geometry, source and detector characteristics and its optical properties which determine the measurement results. By using the fan beam projection method, the projected light distribution of different pixels in the two dimensional plane is uneven. As another necessary feature in image reconstruction, it is essential to derive the sensitivity maps first.

\subsection{Projection Geometry}

The projection geometry of the sensors determines the relationship between the sensors' arrangements and its mathematical modelling. In this research, thirty-two pairs of optical sensors are employed. The optical sensors are divided into thirty-two infrared transmitters and thirty-two photodiode receivers which are placed alternately. These optical sensors are each coupled to separate fibre optics to the periphery of the sensor's fixture. The diameter of the sensor's fixture which mounts the fibre optics is $100 \mathrm{~mm}$ and its circumference equal to $314.2 \mathrm{~mm}$

By distributing the fibre optics evenly around the circumference of the fixture as shown in Fig. (2), the angle between each emitter and its adjacent receiver viewed from the centre of the circle is 5.625 degree (dimensions not to scale in figure). 


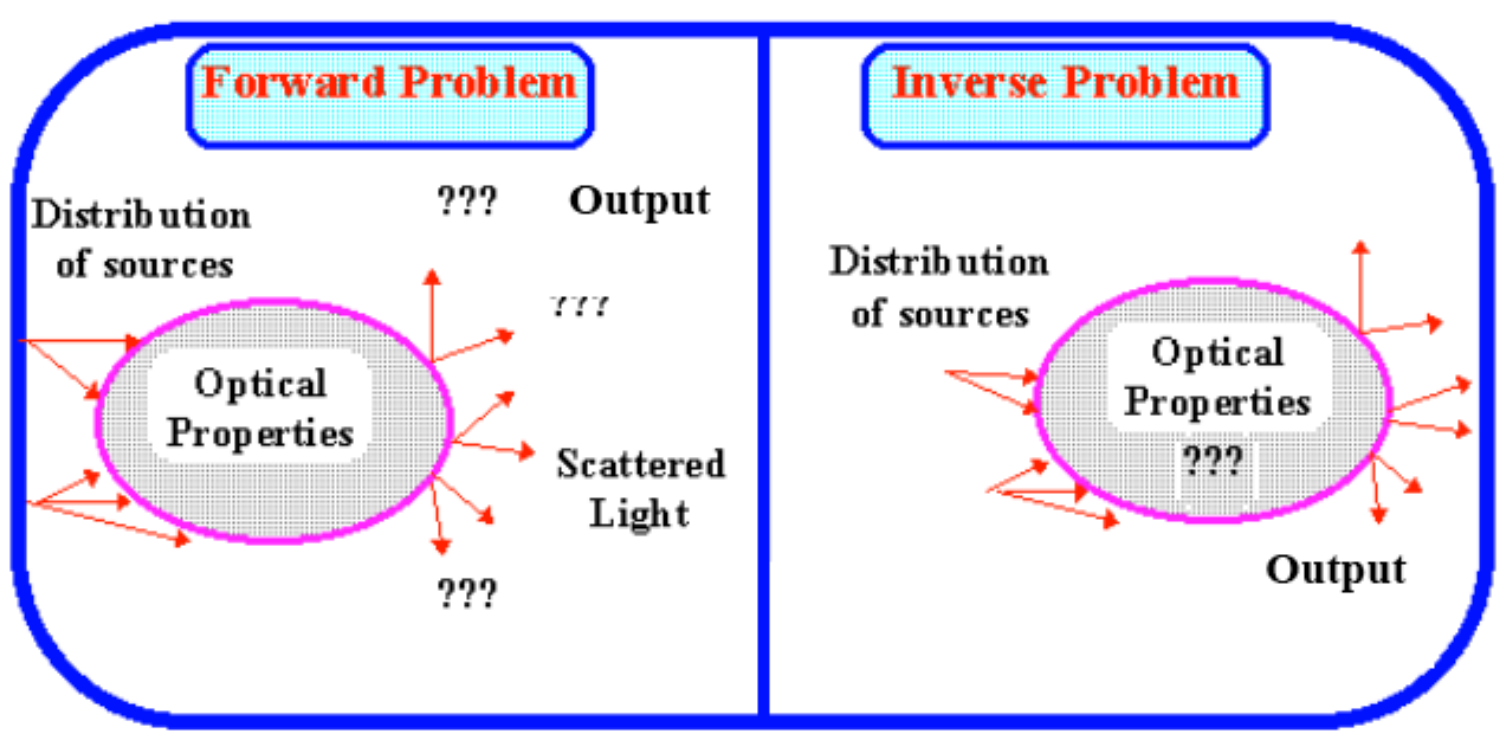

Fig. (1). Illustration of forward problem and inverse problem.

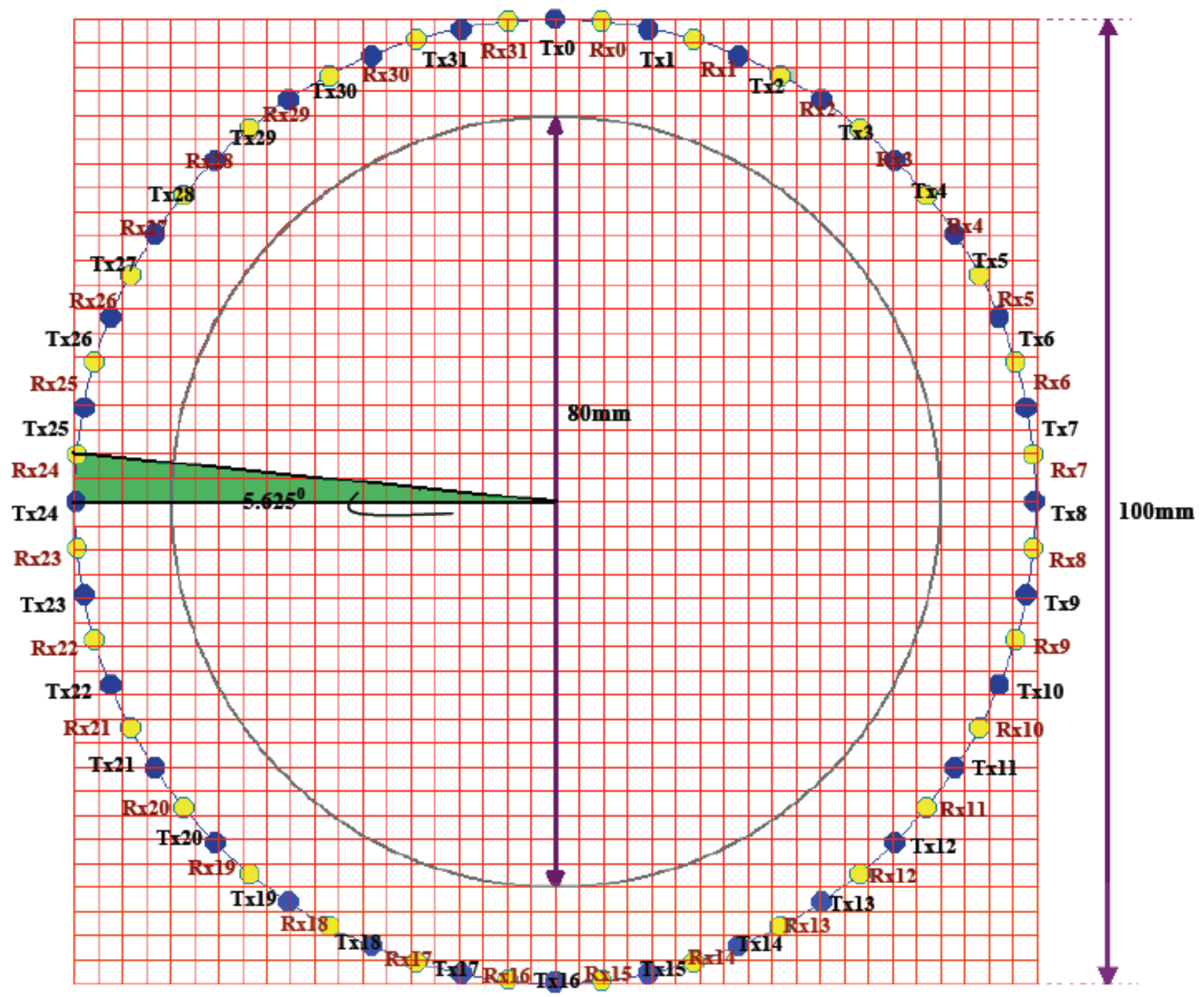

Fig. (2). Optical fibre sensors arrangement on sensor's fixture. 
The optical fibre sensors arrangement in Fig. (2) is seen from the top view. The sensor's fixture is designed to be bigger than the pipeline used. Tx (in blue colour) and $\mathrm{Rx}$ (in yellow colour) represent the respective transmitters and receivers as they are arranged alternately in clockwise direction, starting from Tx0 and ending at Rx31. The $80 \mathrm{~mm}$ dimension is the diameter of the investigated pipe and the red gridlines are the $32 \times 32$ mapping resolution which is applied in solving the forward and inverse problems.
To simplify the mapping of optical fibre sensors to the two-dimension image plane, it is assumed that diameters of both optical fibre sensors (transmitters and receivers) are the same which is $2.20 \mathrm{~mm}$. This assumption is made because the size of the fibre optic's inner core is $1 \mathrm{~mm}$ and after they are lensed, the surface of the inner core has an approximate diameter of $2.20 \mathrm{~mm}$. A two-dimension image plane which is made up of 640x640 pixels in Fig. (3) serves as the mapping platform for the optical fibre sensors.

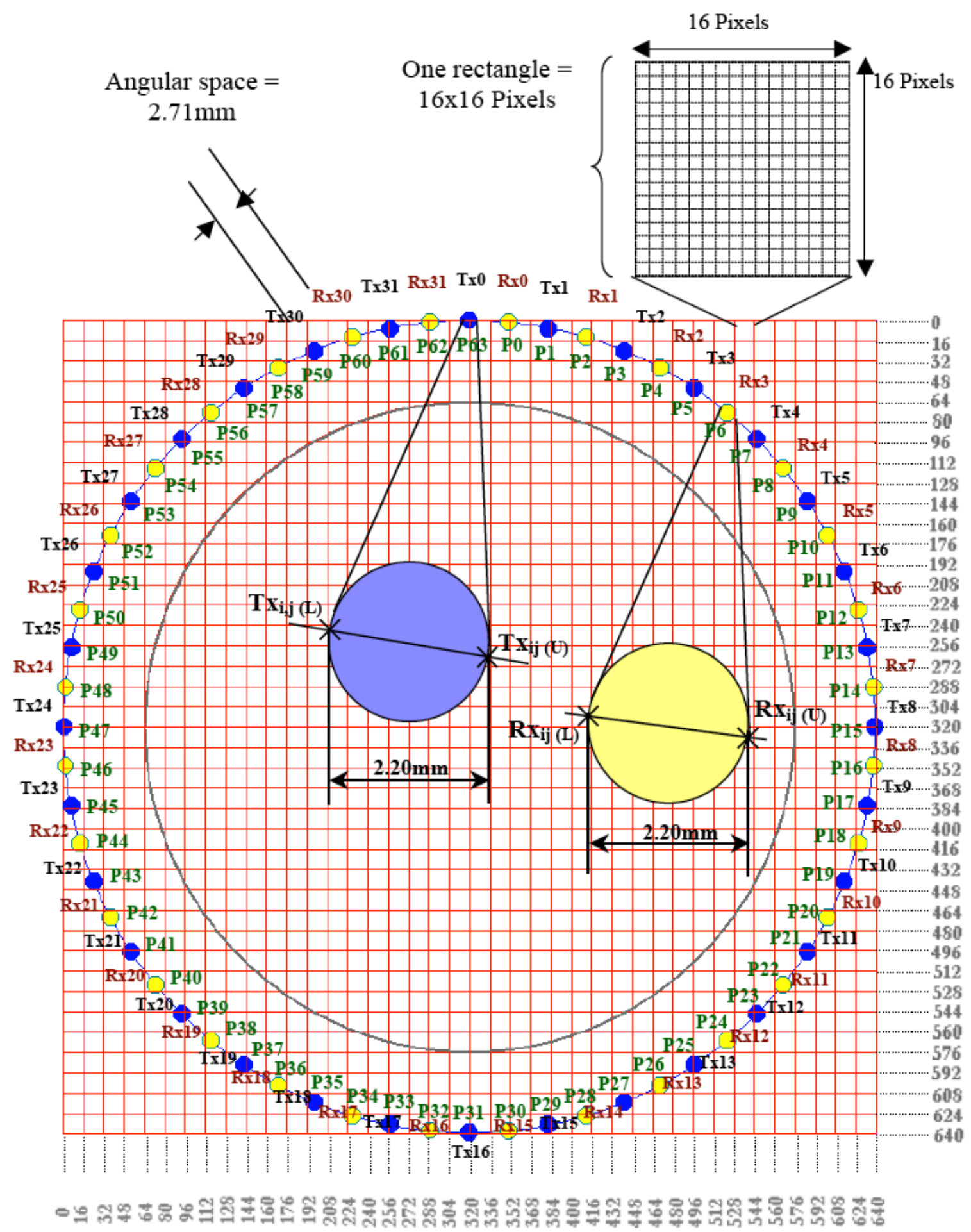

Fig. (3). Optical fibre sensors mapping platform. 
The mapping of sensors on the image plane is important in solving the forward problem. Through Visual $\mathrm{C}++$ programming, the image plane is set to $640 \times 640$ pixels using two-dimensional Cartesian coordinates. The top-left corner coordinate is at $(0,0)$ and the right-bottom end coordinate is $(640,640)$ as shown in Fig. (3). To map the sensors, labelled as P0 to P64, calculations are done by dividing the circle into four quadrants as presented in Fig. (4).

For all the quadrants in Figs. $(\mathbf{3 , 4})$, sensors P0 to P16 can be mapped by using the drawings and equations in Table $\mathbf{1}$, whereby:

$P_{n} \cdot x=$ the x-th coordinate of the n-th sensor.

$P_{n} . y=$ the x-th coordinate of the $\mathrm{n}$-th sensor.

$r=$ the radius is equivalent to 320 pixels. $\theta=$ angle between emitter and its adjacent receiver viewed from the centre of the circle, which is 5.625 degrees.

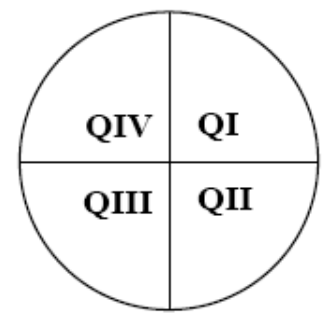

Fig. (4). Dividing the circle into four quadrants.

By using the mapping methods as above, the computer generated coordinates of all the sensors are tabulated in Table 2.

Table 1. Mapping Sensors to Image Plane by Using Cartesian Coordinates

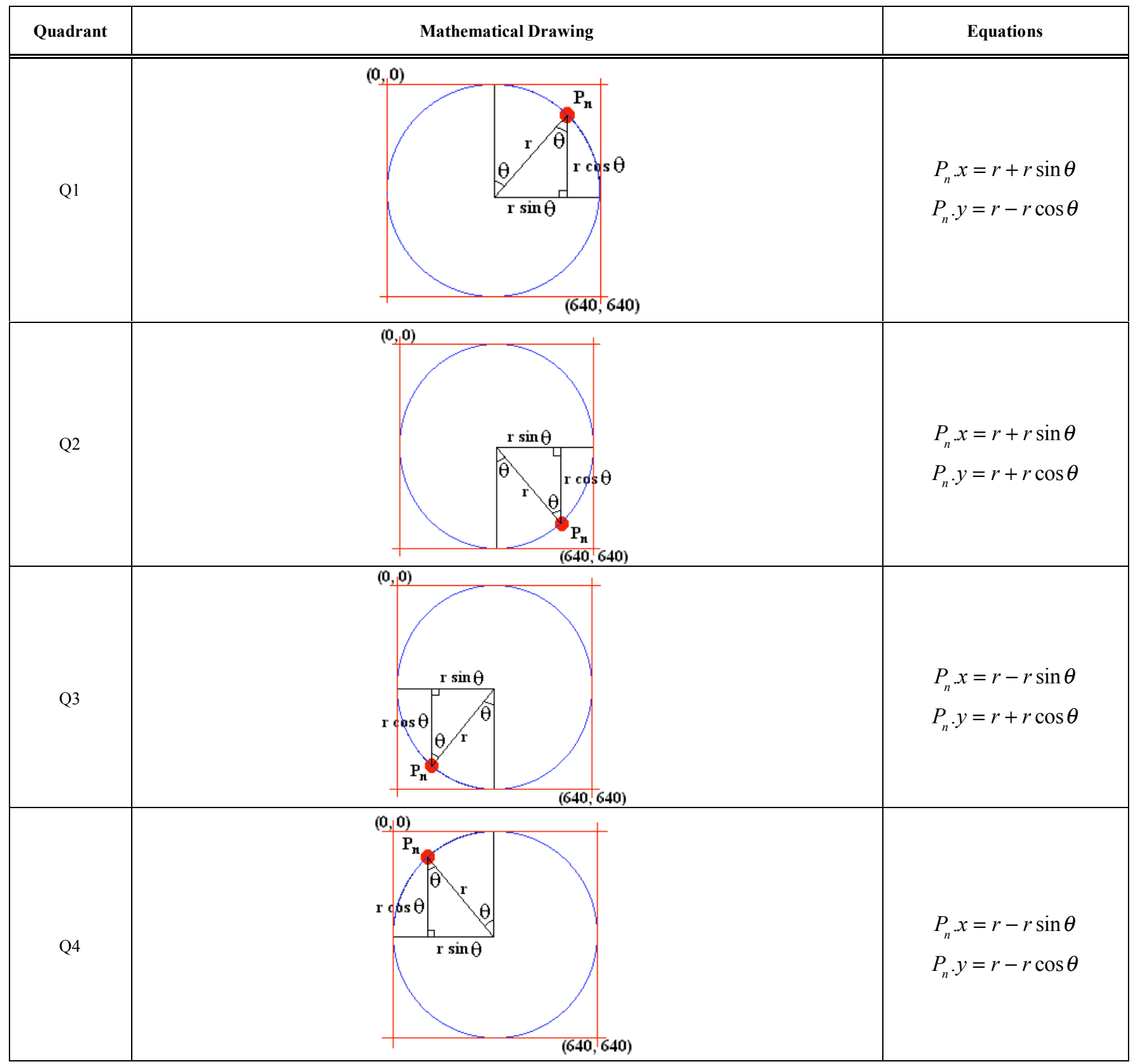


Table 2. The Full Coordinates of All the Sensors from P0 to P63

\begin{tabular}{|c|c|c|c|c|c|c|c|}
\hline Sensor & Coordinate & Sensor & Coordinate & Sensor & Coordinate & Sensor & Coordinate \\
\hline P0 & $(351,2)$ & P16 & $(638,351)$ & P32 & $(289,638)$ & P48 & $(2,289)$ \\
\hline P1 & $(382,7)$ & P17 & $(633,382)$ & P33 & $(258,633)$ & P49 & $(7,258)$ \\
\hline P2 & $(412,14)$ & P18 & $(626,412)$ & P34 & $(228,626)$ & P50 & $(14,228)$ \\
\hline P3 & $(442,25)$ & P19 & $(615,442)$ & P35 & $(198,615)$ & P51 & $(25,198)$ \\
\hline P4 & $(470,38)$ & P20 & $(602,470)$ & P36 & $(170,602)$ & P52 & $(38,170)$ \\
\hline P5 & $(497,54)$ & P21 & $(586,497)$ & P37 & $(143,586)$ & P53 & $(54,143)$ \\
\hline P6 & $(523,73)$ & P22 & $(567,523)$ & P38 & $(117,567)$ & P54 & $(73,117)$ \\
\hline P7 & $(546,94)$ & P23 & $(546,546)$ & P39 & $(94,546)$ & P55 & $(94,94)$ \\
\hline P8 & $(567,117)$ & P24 & $(523,567)$ & P40 & $(73,523)$ & P56 & $(117,73)$ \\
\hline P9 & $(586,143)$ & P25 & $(497,586)$ & P41 & $(54,497)$ & P57 & $(143,54)$ \\
\hline P10 & $(602,170)$ & P26 & $(470,602)$ & P42 & $(38,470)$ & P58 & $(170,38)$ \\
\hline P11 & $(615,198)$ & P27 & $(442,615)$ & P43 & $(25,442)$ & P59 & $(198,25)$ \\
\hline P12 & $(626,228)$ & P28 & $(412,626)$ & P44 & $(14,412)$ & P60 & $(228,14)$ \\
\hline P13 & $(633,258)$ & P29 & $(382,633)$ & P45 & $(7,382)$ & P61 & $(258,7)$ \\
\hline P14 & $(638,289)$ & P30 & $(351,638)$ & P46 & $(2,351)$ & P62 & $(289,2)$ \\
\hline P15 & $(639,320)$ & P31 & $(320,639)$ & P47 & $(1,320)$ & P63 & $(320,1)$ \\
\hline
\end{tabular}

From the $640 \times 640$ pixels, the resolution of the plane is divided into 40x40 rectangles. Each rectangle has a total of 256 pixels. By definition, pixel represents the smallest unit of an image and resolution refers to the clarity or sharpness of an image, initiated by the number of pixels per square inch on a computer-generated display. When the transmitters and receivers are distributed evenly on the periphery of the sensor's fixture, the equispaced interval [2] between each transmitter and the subsequent receiver is $2.71 \mathrm{~mm}$ by using Equation 12.

$$
E . S=\frac{C-(2.20) n}{n}
$$

Whereby:

$E . S=$ Equispaced interval $(\mathrm{mm})$.

$C=$ Circumference (mm), obtained from Equation 3.1.

$n=$ a total of 64 optical fibre sensors.

These pre-determined geometrical co-ordinates and rectangles will be useful in sensor modelling and generating sensitivity maps.

\section{SENSITIVITY MAPS}

From the fan beam projection properties [3], it is known that the fan beam projection can be seen as a point source of radiation that emanates a fan shaped beam. The emanation covers in many directions in different angles. For a given source and detector combination, functions are calculated that describe the sensitivity of each source and measurement pair to changes in optical properties within each pixel of the model [4]. Thus, when the projection beam is mapped onto the two-dimensional image plane, each light beam will spread across each rectangle in different weights as illustrated in Fig. (5).

The solution of forward problem generates a series of sensitivity maps. And the inversion of these sensitivity matrixes therefore provides a reconstruction of the optical properties [4]. Basically, the number of generated sensitivity maps is dependent to the number of projections for the sensors. No matter whether the applied projection method is the 2-projection or 4-projection method, each light beam must be sampled individually. Therefore, for the thirty-two transmitters and the corresponding six receivers which receive light per emission of each transmitter, the total projections are 192. This means that there will be 192 sensitivity maps generated as well. Before these sensitivity maps can be used for image reconstruction, they must be normalized first.

Normalization of sensitivity maps is done when there is no simple linear relationship between the weight distributions in a rectangle for different light projections. For example, the rectangle $(25,30)$ might have certain percentage of light passing through for the light beam of transmitter 10 and receiver 25 . When transmitter 0 emits light to receiver 16 , this light beam might pass through rectangle $(25,30)$ with a different weight distribution. If there are other light beams passing through the same pixel as well, the weight distribution of the pixel will become complicated. Thus, it is necessary to build a general relationship between the pixels and all the projection beams that pass through the pixels. A simple approach to normalize the rectangle values in this research is engaged in a similar manner as normalizing the pixel values in ECT so that they have the values of zero and one when the rectangle contains the lower and higher weight distributions respectively. 


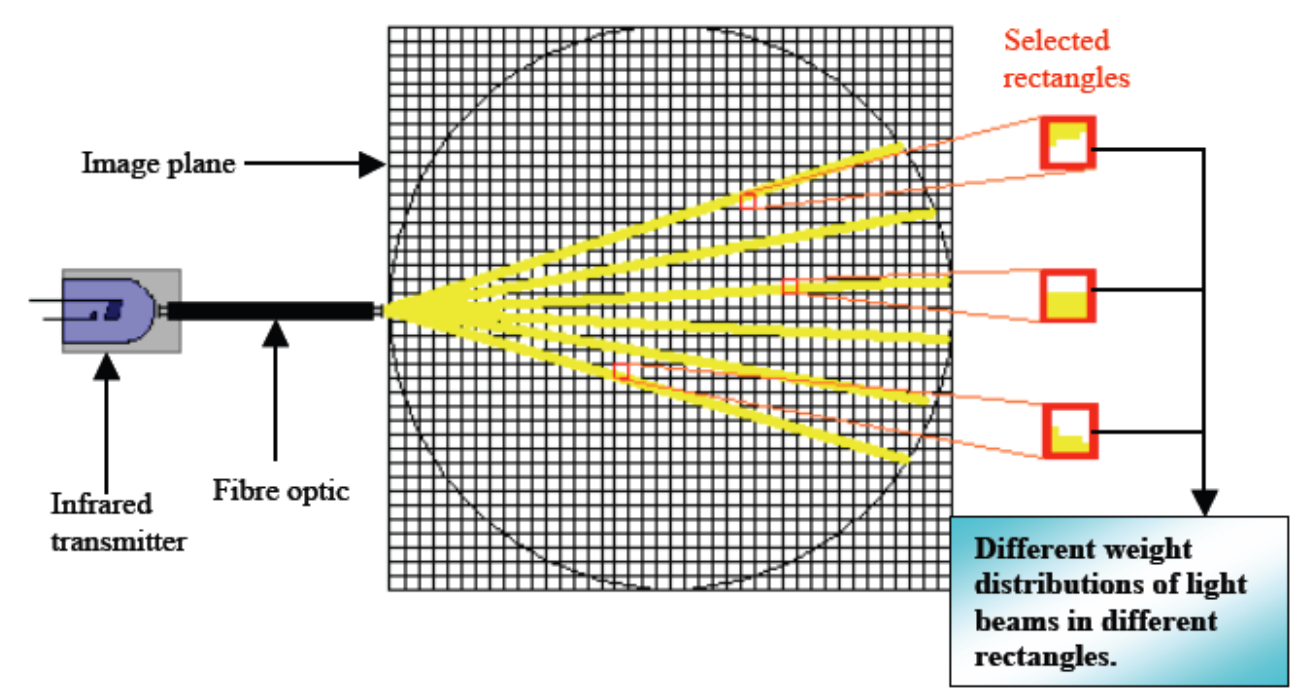

Fig. (5). Fan beam projection map.

As a reference to map the sensitivity maps onto the 2dimension image plane, Fig. (3) is being referred. From the $640 \times 640$ pixels plane, there are gridlines to divide the pixels into $40 \times 40$ rectangles where each rectangle contains $16 \times 16$ pixels. In image reconstruction, the actual area of flow is from pixel 64 to pixel 575 in $x$-axis and y-axis. In this section and the following chapters, the image plane is reconfig- ured so that all calculations and scanning of pixels are done only for the part of the actual flow area. This is being done to avoid wasting unnecessary time and resources used to calculate and scan the whole $640 \times 640$ pixels. The resolution of the actual image plane thus becomes $32 \times 32$ in image reconstruction which contains $512 \times 512$ pixels. This explanation can be further explained graphically in Fig. (6).

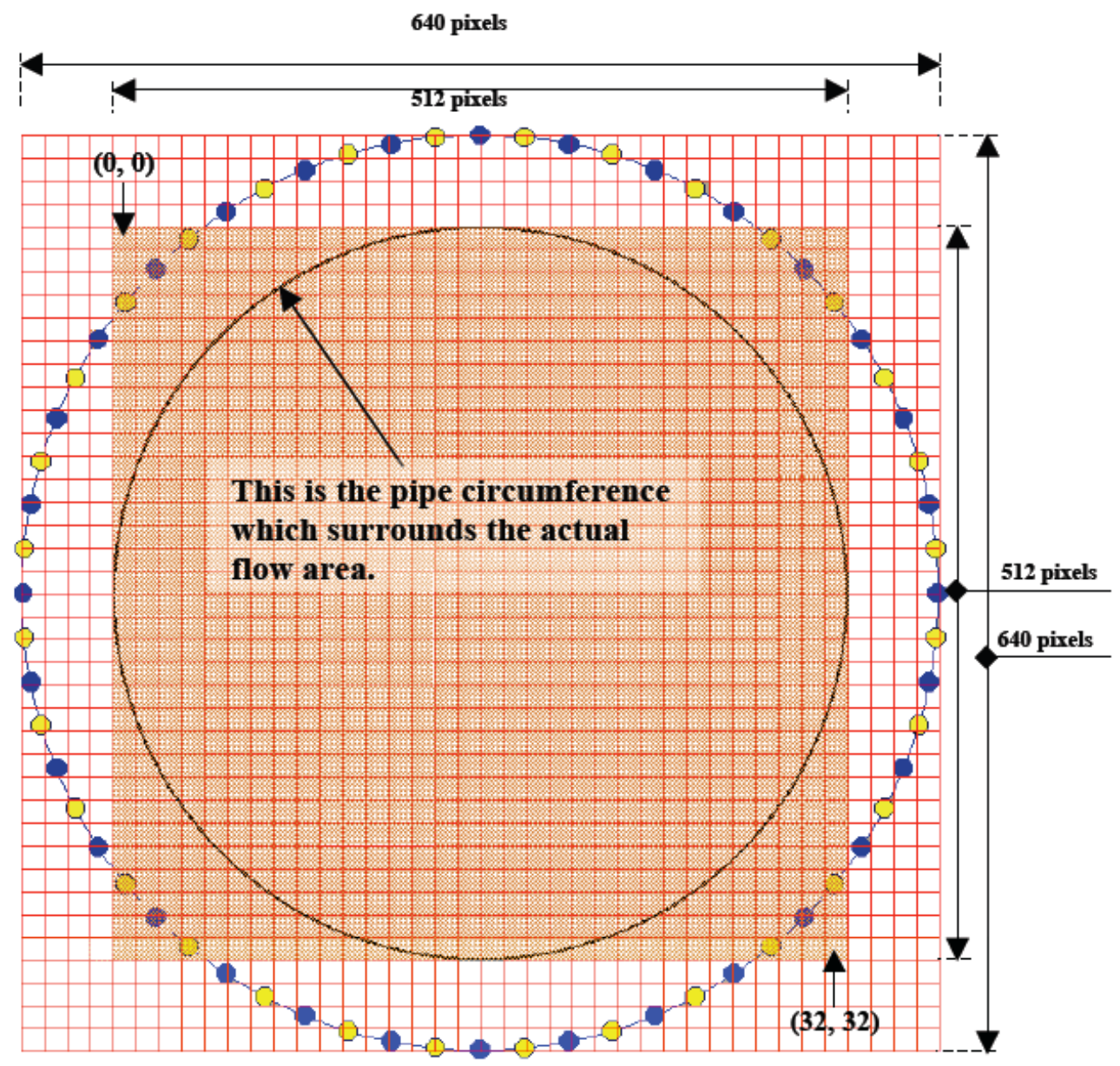

Fig. (6). The actual flow image plane. 
After the actual flow area is being identified, the sensitivity maps are generated using Visual $\mathrm{C}++$ programming language as shown in Equation 2.

$$
S_{i, j}(x, y)=\sum_{a=0}^{511} \sum_{b=0}^{511} P_{x, y}(a, b)\left\{\begin{array}{l}
P_{x, y}(a, b)=1 ; \text { if black= changed } \\
P_{x, y}(a, b)=0 ; \text { if } \text { white = unchanged }
\end{array}\right.
$$

Whereby:

$S_{i, j}(x, y)=$ sensitivity map of light beams for all transmitters and receivers.

$P_{x, y}(a, b)=$ an array to represent the total black pixels in rectangle-xy.

$a$ and $b=$ the $a$-th column and $b$-th row of the pixels in the actual flow plane.

$x$ and $y=$ the $x$-th column and $y$-th row of the $32 \times 32$ rectangles or resolution.

Next is to sum and normalize all the 192 sensitivity maps using the following two approaches:

i. Summing the maps according to the weights of the same rectangles from all projections and normalizing the maps (rectangle-based normalization).

ii. Summing the maps based on weights of all the rectangles for individual light projection and normalizing the maps (projection-based normalization).

The rectangle-based normalization is to total up all the total black pixels from all different projections in a same rectangle using Equation 3 and normalize the maps according to Equation 4.

$$
\begin{aligned}
& T 1(x, y)=\sum_{i=0}^{32} \sum_{j=0}^{16} S_{i, j}(x, y) \quad\left\{\begin{array}{l}
0 \leq x<32 \\
0 \leq y<32
\end{array}\right. \\
& N 1_{i, j}(x, y)=\left\{\begin{array}{lr}
\frac{S_{i, j}(x, y)}{T 1(x, y)} & ; \text { for } T 1(x, y)>0 \\
0 & ; \text { for } T 1(x, y)=0
\end{array}\right.
\end{aligned}
$$

whereby:

$T 1(x, y)=$ the total or sum of the same element in rectanglexy obtained from the

192 sensitivity maps.

$S_{i, j}(x, y)=$ the 192 sensitivity maps.

$N 1_{i, j}(x, y)=$ the normalized sensitivity maps for light beams of all $\mathrm{Tx}(0 \leq i<32)$ to $\mathrm{Rx}(0 \leq j<6)$ using the rectanglebased normalization.

Meanwhile, by using the projection-based normalization, the total weights of pixels for all rectangles (32 rectangles) for each light projection is being summed by using Equation 5 and then normalized as stated in Equation 6.

$T 2(i, j)=\sum_{x=0}^{32} \sum_{y=0}^{32} S_{i, j}(x, y)$
$N 2_{i, j}(x, y)=\frac{S_{i, j}(x, y)}{T 2(i, j)} \quad\left\{\begin{array}{l}T 2(i, j)>0 \\ 0 \leq i<32 \\ 0 \leq j<6\end{array}\right.$

Whereby:

$T 2(i, j)=$ the total of pixel weights for all rectangles in individual light projection.

$S_{i, j}(x, y)=$ the 192 sensitivity maps.

$N 2_{i, j}(x, y)=$ the normalized sensitivity maps for light beams of all $\mathrm{Tx}$ to $\mathrm{Rx}$ using the projection-based normalization.

Since the normalized maps generated in Equation 4 and Equation 6 has a total of 192 maps each.,

\section{INVERSE PROBLEM}

The solution to the inverse problem involves the process of combining the computed sensitivity maps with the measured data (sensors' readings) to obtain an image of the particle concentration distribution within the conveyor [5]. In other words, consider that a sensor's array giving us the projection data, the inverse problem involves in estimating the original tracer distribution $\mathbf{f}(\mathbf{x}, \mathbf{y})$ as illustrated in Fig. (7).

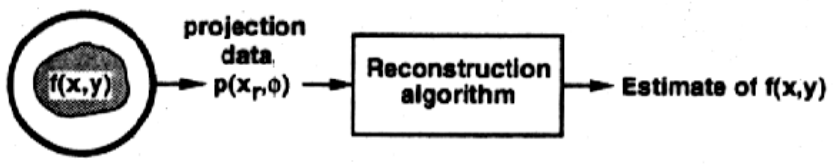

Fig. (7). The basic idea of solving the inverse problem.

The works done by previous researchers in industrial optical tomography such as Ruzairi [6], Sallehuddin [7] and Chan [8] mainly focused on applying the back projection algorithms to construct the cross-sectional images. This paper will focus on computing and comparison analysis of cross-sectional image using the two most basic but dissimilar algorithms which are the linear back projection (LBP) and iterative reconstruction algorithms (IRA). As we can see, the LBP differs with IRA in the sense that the former uses a linear relation to solve the inverse problem while the latter treats the inverse problem in a non-linear fashion, by using an iterative method.

\subsection{Linear Back Projection Algorithm (LBP)}

Most work in process tomography has concentrated on the use of a linearised version of the back-projection algorithms originally developed for X-ray tomography. These have the advantages of low computation cost, simple and have a fast computational time which is capable of real-time operation in many applications [9]. In implementing the back projection algorithm, the measurements obtained at each projection are projected backwards along the same line in the image space, assigning the measured value to each point in the line as described by Chan [8] using Fig. $(\mathbf{8 a}, \mathbf{b})$.

This crude back projection in Fig. (8b) assumes equal probability that the object contributing to a point on the projection lay at any point along the ray producing the point. To obtain the density distribution of the image at any point is to sum the attenuation coefficients of the rays along a straight 
line. Ruzairi [6] describes the acquisition of density distribution in back projection process using Equation 7.

(a)

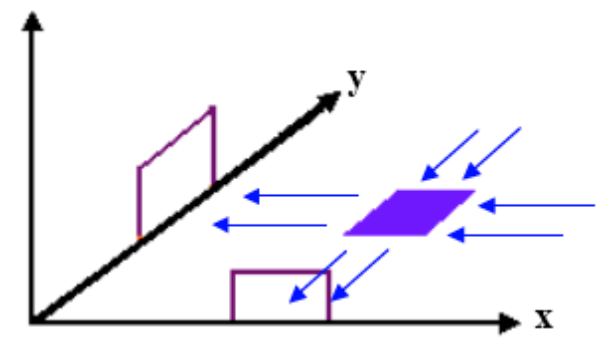

(b)

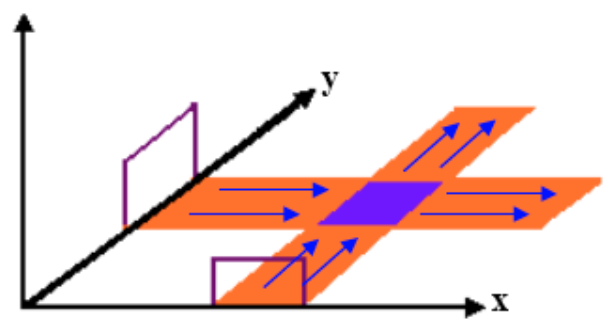

Fig. (8). Implementing back projection algorithm in image space (Chan, 2002a) (a) Projection (b) Back projection.

$$
f(x, y)=\sum_{j=1}^{m} P_{j}\left(x \cos \theta_{j}+y \sin \theta_{j}\right) \Delta \theta
$$

which is the general case for $m$ projections, whereby:

$f(x, y)=$ the function of reconstructed image from back projection algorithm.

$\theta_{j}=$ the $\mathrm{j}$-th projection angle.

$\Delta \theta=$ the angular distance between projections and the summation extends over all the $m$ projections.

The Linear Back Projection (LBP) is based on first obtaining the sensitivity distributions (or sensitivity maps) for all sensor pairs and then linearly superimposing the normalized sensor readings using the sensitivity maps as the weighting factors to obtain images [10]. In the parallel beam projection, the application of LBP to obtain the density distribution of an object is simple. Fig. (9) shows an object $\mathrm{R}$ located inside the pipe which results in the overlapping of pixels when generated by back projections of rays.

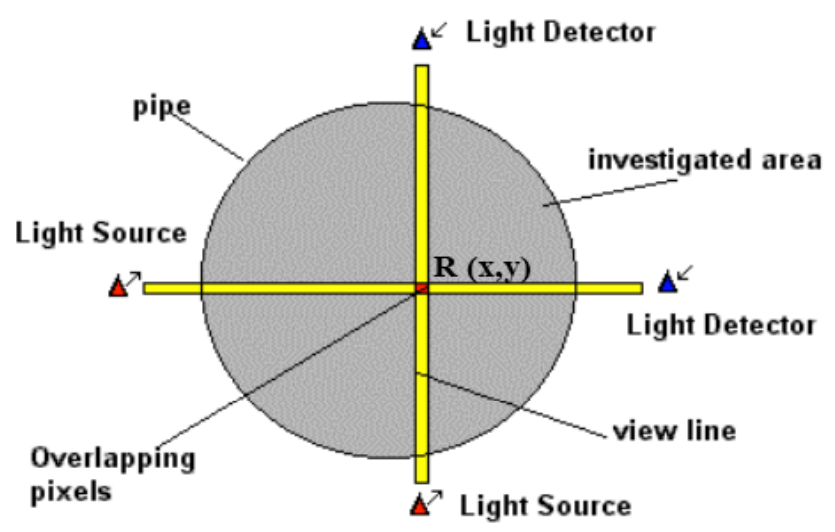

Fig. (9). Overlapping of pixels using LBP for parallel beam.
For parallel projection, each sensor corresponds to one sensitivity map. Pixels outside the pipe will not contribute to the measurement so the sensitivity value is zero. If the beam of the sensor is being considered passing through a pixel within the pipe, the pixel has a sensitivity of one, otherwise zero. Based on this algorithm, the sensor voltage is multiplied with its sensitivity matrix (in this case, sensitivity is 1 or 0). From Fig. (9), the concentration value for the pixel $\mathrm{R}(x, y)$ can be obtained by simplifying Equation 7 into a new equation as shown in Equation 8,

$V_{x, y}=V_{x}+V_{y}$

whereby,

$V_{x, y}=$ Voltage distribution profile (concentration profile).

$V_{x}=$ Voltage produced for $x$-th sensor.

$V_{y}=$ Voltage produced for $y$-th sensor.

For the LBP that is implemented in this research, the calculation is slightly more complicated than the calculation used in parallel beam. As discussed earlier in solving the forward problem, the fan beam projection has different sensitivity maps for each combination of transmitters and receivers. Fig. (10) illustrates the LBP concept in fan beam projection in a simple way.

In Fig. (10a), the ray-sum resulting from a single dense object is back-projected so that it is distributed homogeneously throughout the same line in which the measurement of the ray-sum is made. As different projections are formed, the dense object is identified within its orientation of the projections. Next in Fig. (10b), it shows that the individual back projections are summed. According to the basic theory to obtain image reconstruction, the LBP algorithm combines each sensor reading with its sensitivity map to generate the concentration profile within the pipeline [1]. The sensitivity map for fan beam projection cannot be simplified to the binary values of ' 0 ' and ' 1 ' but each sensitivity map has to be multiplied accordingly with the sensor readings. The same elements of these matrices are summed to provide the back projected voltage distributions as expressed mathematically in Equation 9 [11].

$V_{L B P}(x, y)=\sum_{i=0}^{32} \sum_{j=0}^{6} \hat{V}_{j, i} * N 1_{i, j}(x, y)$

whereby:

$V_{L B P}(x, y)=$ the voltage distribution (concentration profile in unit Volt) using LBP

algorithm in $n \times n$ matrix whereby $n$ is equivalent to the sensitivity

map's dimension.

$\hat{V}_{j, i}=$ the signal loss amplitude of $\mathrm{j}$-th receiver for $\mathrm{i}$-th projection in unit Volt.

$N 1_{i, j}(x, y)=$ the normalized sensitivity maps for light beams of all transmitters to receivers using the rectangle-based normalization. 


\section{Transmitters}

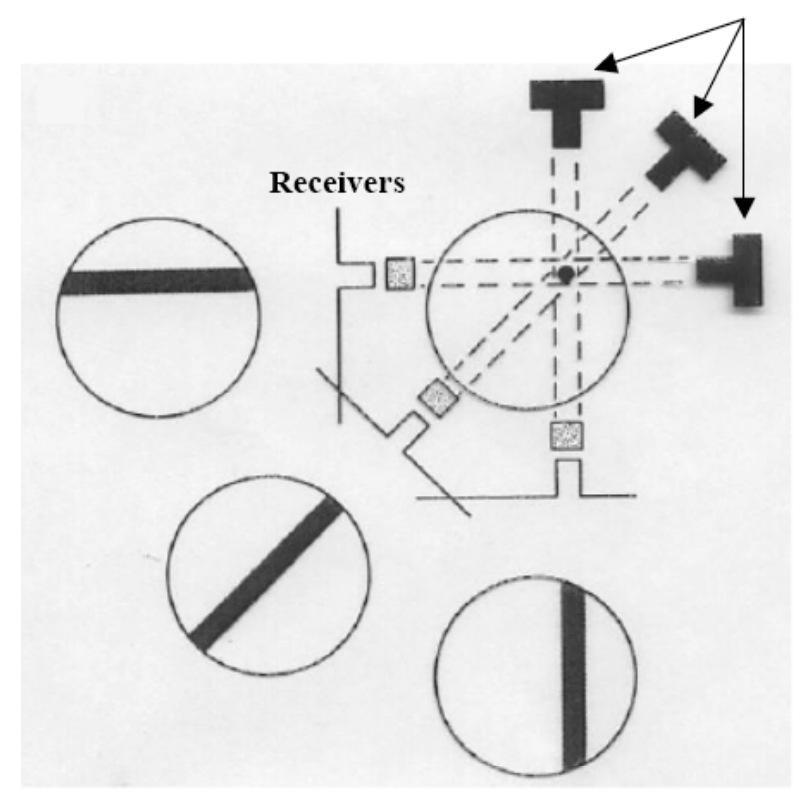

(a)

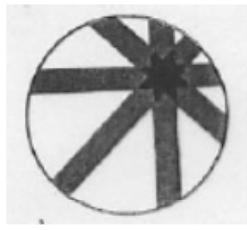

(b)

Fig. (10). LBP concept in fan beam projection (a) Different back projected rays resulted from fan beam emanation (b) Ray summation for fan beam projections.

From Equation 9, we can see that $\hat{V}_{j, i}$ represents the sensor loss and not the output sensor values obtained directly from the signal conditioning circuits. Basically, the output signals from the sensors depend on the designed signal conditioning circuits. The standard LBP algorithm requires multiplication of sensitivity maps with voltage readings which is proportional to the flow rate. Since the signal conditioning circuit in this project results in proportional signal outputs to the received lights instead of the flow rate, the sensor values must be normalized first. The fan beam projection in optical tomography faces the same problem as faced by ECT systems. Same as ECT systems, fan beam projections have relatively large number of different measurements required and the fact that the relationship between different projections and the non-uniform signals received by sensors for each of these measurements, creating potential calibration and operating problems.

To overcome both the non-uniform signal and normalization of sensor value issues in real-time flow measurement, calibration of sensors are done before any measurements are commenced which means that the non-flow sensor output is stored as a reference voltage. This reference voltage will be known as the calibration voltage and will be used in obtaining the signal loss amplitude for image reconstruction using the LBP algorithm as referred to in Equation 10.

$\hat{V}_{j, i}=$ Vcal $_{j, i}-V_{j, i}$

whereby:

$\widehat{V}_{j, i}=$ the signal loss amplitude of $\mathrm{j}$-th receiver for $\mathrm{i}$-th projection in unit Volt.
$\operatorname{Vcal}_{j, i}=$ calibration or reference signal of $\mathrm{j}$-th receiver from i-th projection.

$V_{j, i}=$ received signal of $\mathrm{j}$-th receiver from $\mathrm{i}$-th projection.

By using the output signal normalization in Equation 10, the output voltage readings which are proportional to the flow rate can thus be obtained and this fulfils the requirement of the general LBP algorithm.

\subsection{Iterative Reconstruction Algorithn (IRA)}

A great demand for high quality images generated by ECT has led many researchers to develop reconstruction techniques based on iterative algorithms [12] [10] has emphasized that iterative methods can produce much better images than non-iterative construction methods for ECT systems. Apart from ECT, the iterative reconstruction method plays a substantial role for a better image quality when applied in medical imaging using Computerized Tomography or CT [13]. With its benefits proclaimed in ECT and medical imaging, it is indeed a motivation to apply the IRA method in the Optical Fibre Process Tomography (OFPT).

Generally, the iterative reconstruction is an algorithm which iteratively updates an estimate of the image until raysums or projection measurements computed from the image match adequately the input projection measurements. There are a variety of algorithms, and each starts with an assumed image, computes projections from the image, compares the computed projections with the original measurements and updates the image based upon the difference between the calculated projections and the actual measurements. Iterative algorithm has the advantage that a-priori information [14] can be incorporated at each step of the reconstruction proc- 
ess. For example, one can use knowledge that the object density cannot be negative and is zero outside of a defined boundary to reduce image artifacts.

The idea of the IRA approach in this paper is to use the forward and inverse transforms alternately to progressively correct the pixel values, and is based on the assumption that the forward transform is reasonably accurate if the field distortion is low but that the inverse transform may be very inaccurate. This technique is conceptually similar to the practice of correcting the distortion of an imperfect amplifier by the use of negative feedback. The steps to perform the iterative image reconstruction technique [15] are simplified as follows:

a) Take a set of measurements and normalize a set of 192 sensor readings, $\hat{V}_{(1)}$ for one image frame.

b) Obtain the concentration profile, $V_{L B P(1)}$ for the measured sensor values in step (a) using the sensitivity map, $N$ obtained in (the inaccurate) forward transform which has been normalized using rectangle-based normalization.

$V_{L B P(1)}=N \cdot \hat{V}_{(1)}$

a) The approximate concentration values obtained in step (b), $V_{L B P(1)}$ are then used to back-calculate a set of sensor values, $\hat{V}_{(2)}$ using the (relatively accurate) inverse transform. Note that the normalized sensitivity map here, $N^{T}$ is being normalized using the projection-based normalization.

$\hat{V}_{(2)}=N^{T} \cdot V_{L B P(1)}$

b) A set of error sensor values, $\Delta V$ are calculated and truncated to limit the maximum values of $\Delta \hat{V}$ so that they lie within a range of $(-0.05<\Delta \hat{V}<0.05)$. This is necessary to prevent the feedback loop from being unstable.

$\Delta \hat{V}=\hat{V}_{(2)}-\hat{V}_{(1)}$

c) The error sensor values, $\Delta V$ is used to generate a set of error concentration profile, $\Delta V_{L B P}$.

$\Delta V_{L B P}=N \cdot \Delta \widehat{V}$

d) This error concentration profile, $\Delta V_{L B P}$ is then used to correct the previous set of concentration profile to generate a new set of concentration values, $V_{L B P(2)}$.

$V_{L B P(2)}=V_{L B P(1)}-\Delta V_{L B P}$

e) Display image.

f) Repeat steps (c) to (g) using the new set of $V_{L B P}$ values, $V_{L B P(2)}$ (instead of $V_{L B P(1)}$ ) in Equation 11. In Equation 13, generate the error sensor values by subtracting the original measured sensor values from the current set.

g) Repeat step (h) as many times as necessary to obtain an accurate image.

Basically, the first two steps (step (a) and step (b)) are equivalent to the normal LBP algorithm approaches to obtain the concentration profiles $\left(V_{L B P}(x, y)\right)$. From here, the error measurements are iterated until the concentration values converge to the correct solution. It is possible to improve the image resolution and accuracy to values much closer to the theoretical limit by the use of iterative techniques.

\section{RESULT \& DISCUSSION}

\subsection{Real-Time Concentration Measurements}

In previous section, the feasibility of the image reconstruction algorithms are being investigated by comparing the reconstructed images generated from the predicted sensor values in sensor modelling using both the LBP and iterative reconstruction algorithms. In this section, the feasibility of the designed sensors is being tested for the static and dynamic flows. For the static flow experiments, stationary objects (the objects as referred to in Table 3 ) are placed inside the sensing area.

Through forward modelling, the object diameters in unit pix or pixels are drawn onto the image plane. This represents the true distribution of the object. By predicting the sensor values during object disruption, and also using the LBP and iterative algorithms, the tomograms for the modelled images are generated. A range of iteration numbers is processed: one, five, ten, fifteen, twenty, twenty-five and thirty. Zero iteration represents the result of LBP algorithm, and at the same time verifies that the iterative reconstruction algorithm can only be obtained after the LBP algorithm is implemented. In other words, the iterative reconstruction algorithm is the further process of the LBP algorithm data

This means that the concentration measurements and tomograms of the real-time image obtained using the hardware system is being compared with the reconstructed images generated. For the image quality comparison, it investigates how reliable the constructed optical fibre sensor's

Table 3. Properties of Single Flow Objects

\begin{tabular}{|l|c|c|c|c|c|}
\hline \multicolumn{1}{|c|}{ Object } & Shape & Diameter (mm) & Area (mm²) & Object Diameter (pix) & Object Area (pix $\left.{ }^{2}\right)$ \\
\hline \hline A. Percision screwdriver & Round & 2.50 & $4.9 \mathrm{~mm} 2$ & 16 & 200 \\
\hline B. Wire & Round & 1.10 & $3.8 \mathrm{~mm} 2$ & 7.04 & 156 \\
\hline C. Wire-wrap & Round & 0.45 & $0.64 \mathrm{~mm} 2$ & 2.88 & 26 \\
\hline
\end{tabular}


hardware is in producing an image which should be close to the reconstructed image obtained from sensor modelling.

Meanwhile, for the dynamic flow experiments, a miniature pipeline is being constructed to monitor the feasibility of the designed sensors to measure bulk flows. This experiment is essential to before proceeding to obtain the mass flow rate solid/gas flow using the gravity drop conveyor. If the sensors are able to monitor different concentrations of plastic beads flow in real-time, the designed sensor is thus ready to be applied in obtaining mass flow rate of the plastic beads flow. Both the static and dynamic flow experiments are being carried out in room temperature of approximately 25 degrees Celsius.

\subsection{Static Flow Experiments}

In sensor modelling [16], the placing of the investigated object does not face the problem of alignment because there are gridlines which are drawn using programming method. However, in the hardware, there are no gridlines to assist the placing of the objects, specifically the single object and multiple objects flow model. To solve this problem, a piece of polystyrene is being cut according to the size of the flow regime in the sensor's area. On the polystyrene, gridlines are drawn approximately similar to the gridlines in programming. Then, the objects are inserted through the polystyrene according to the proposed locations.

From the summary obtained in static distribution flow models, the tomogram images that will be compared here are the single objects and multiple objects flow images recon- structed by LBP algorithm and also the iterative algorithm at the average optimized level of $10^{\text {th }}$ iteration. The Spatial Image Rrror (SIE) and Peak Signal to Noise Ratio (PSNR) error measurements are also calculated for analysis purposes. Fig. (11) shows the comparison of images obtained. The colour bars used to represent the range of colour gradients for the tomograms are as shown below the numbering for each row.

The SIE and PSNR error values for the reconstructed tomograms are presented in Table $\mathbf{4}$ and then graphically plotted in Fig. (12).

Generally, the graphs for all the flow objects show small values of the SIE for either the LBP (0 iteration) or the iterative reconstruction algorithm at $10^{\text {th }}$ iteration. The range for the SIE error is just in between 0 to 2.5 and is considered as small and acceptable. All the SIE values for the $10^{\text {th }}$ iteration tend to be higher than the SIE values for LBP image. The average value of PSNR is quite high, between the ranges of $50 \mathrm{~dB}$ to $70 \mathrm{~dB}$. The small SIE but high PSNR values concludes that the constructed optical fibre sensors are reliable to produce images which is close to the images reconstructed from predicted sensor values.

\subsection{Dynamic Flow Experiments}

As stated earlier, the aim of carrying out the dynamic flow experiment is to investigate the feasibility of the optical fibre sensors in visualizing bulk solid/gas flow. In these experiments, a series of filters which comprise of $10 \%, 20 \%$, $30 \%, 40 \%, 50 \%, 60 \%, 70 \%, 80 \%$ and $90 \%$ of the total concentration of the investigated $80 \mathrm{~mm}$ diameter pipeline is

Table 4. Assessment of Error Analyses for Reconstructed Images

\begin{tabular}{|c|c|c|c|c|c|c|}
\hline Error & Iteration & \multicolumn{5}{|c|}{ Flow Models } \\
\hline SIE & 10 & 0.736 & 0.966 & 2.117 & 1.211 & 0.758 \\
\hline PSNR & 0 & 57.591 & 63.086 & 56.785 & 53.338 & 52.771 \\
\hline
\end{tabular}

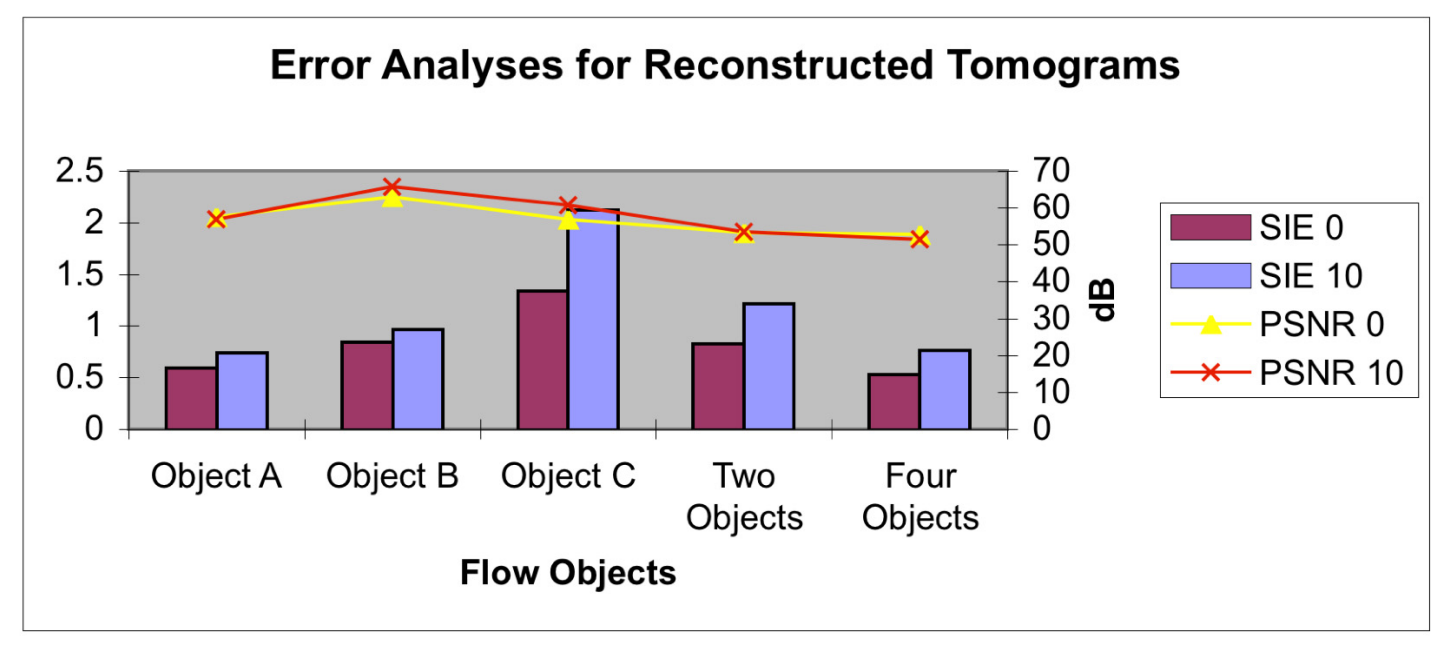

Fig. (11). SIE and PSNR error analyses for reconstructed images. 
being used to limit the plastic beads flow. From a distance of $12 \mathrm{~cm}$ from the interchangeable concentration filter, the optical fibre sensors is expected to be able to measure the filtered flow as the plastic beads flow through the sensor's area. The miniature flow rig diagram is shown in Fig. (12).
The filters used are made of strong cardboard and the shapes of the ellipse opening of the filters are sized according to the area (representing concentration) ratio calculation using Visual $\mathrm{C}++$ programming. Using the top pipeline as a tank, 1500 grams of plastic beads are flows down through the filters before passing through the sensor's area once the

(a)

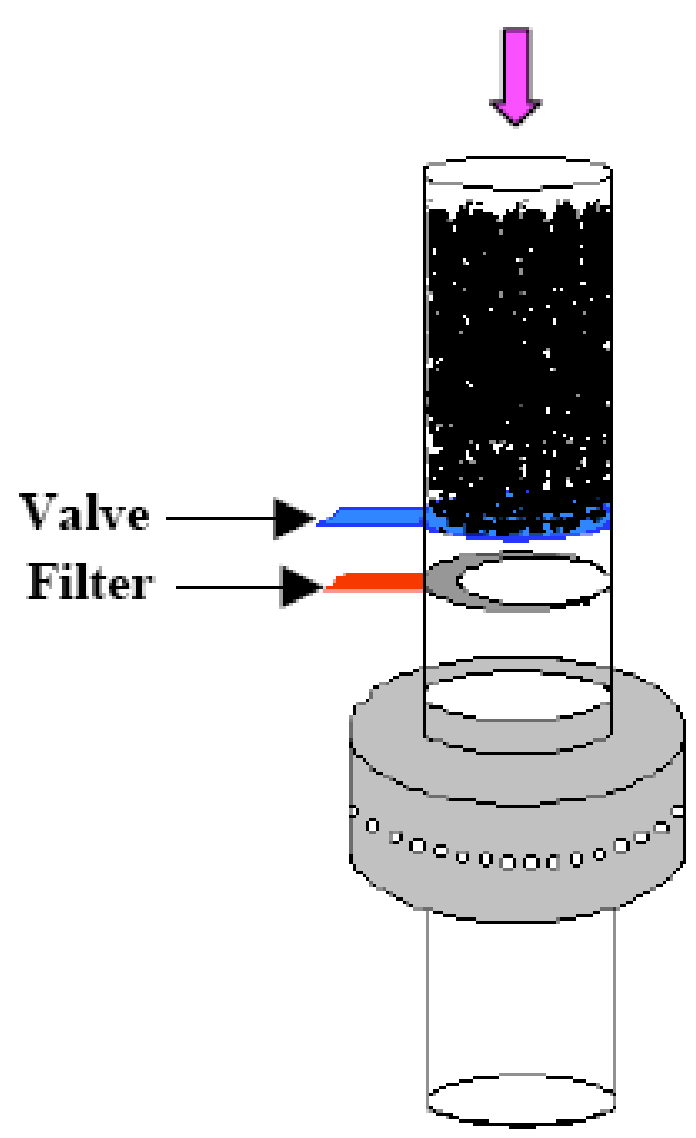

(b)

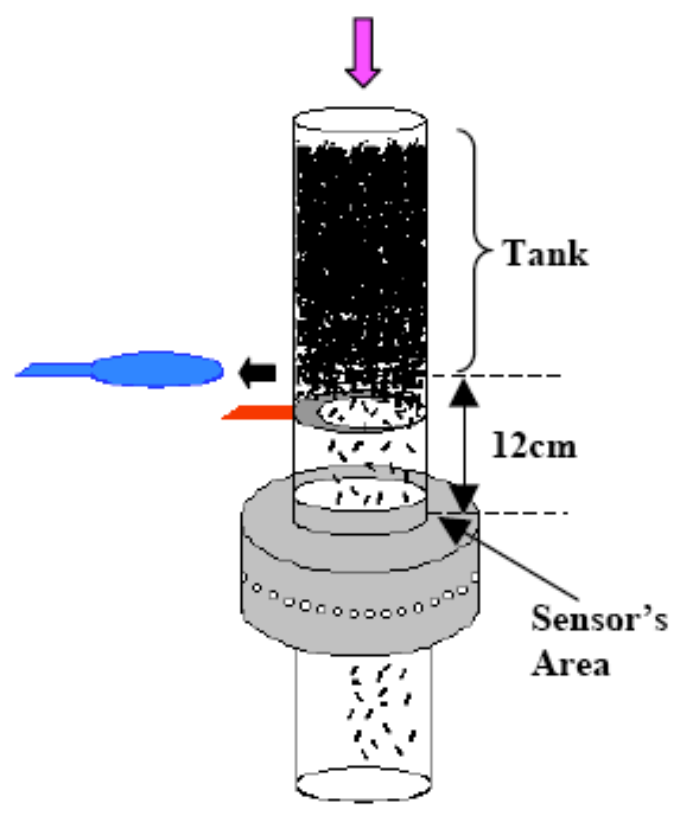

Fig. (12). Miniature flow rig for dynamic flow experiment (a) Valve at 'close' position / non-flow (b) Valve at 'open' position. 
valve is opened. The voltage loss obtained from the optical fibre sensors will be acquired and sent to the computer for image processing and display. The tomogram images are being reconstructed using the LBP and optimized iterative reconstruction algorithm at $10^{\text {th }}$ iteration. The tomogram results for the real-time plastic beads flow is shown in Fig. (13).

\begin{tabular}{|c|c|c|c|}
\hline$\%$ flow & $\begin{array}{ll}\text { Filter } \\
\end{array}$ & LBP & $10^{\text {th }}$ Iteration Image \\
\hline 10 & $\bigcap_{\text {HIGH }}$ & & \\
\hline 30 & & & \\
\hline 40 & $\square_{\text {Low }}$ & & \\
\hline
\end{tabular}

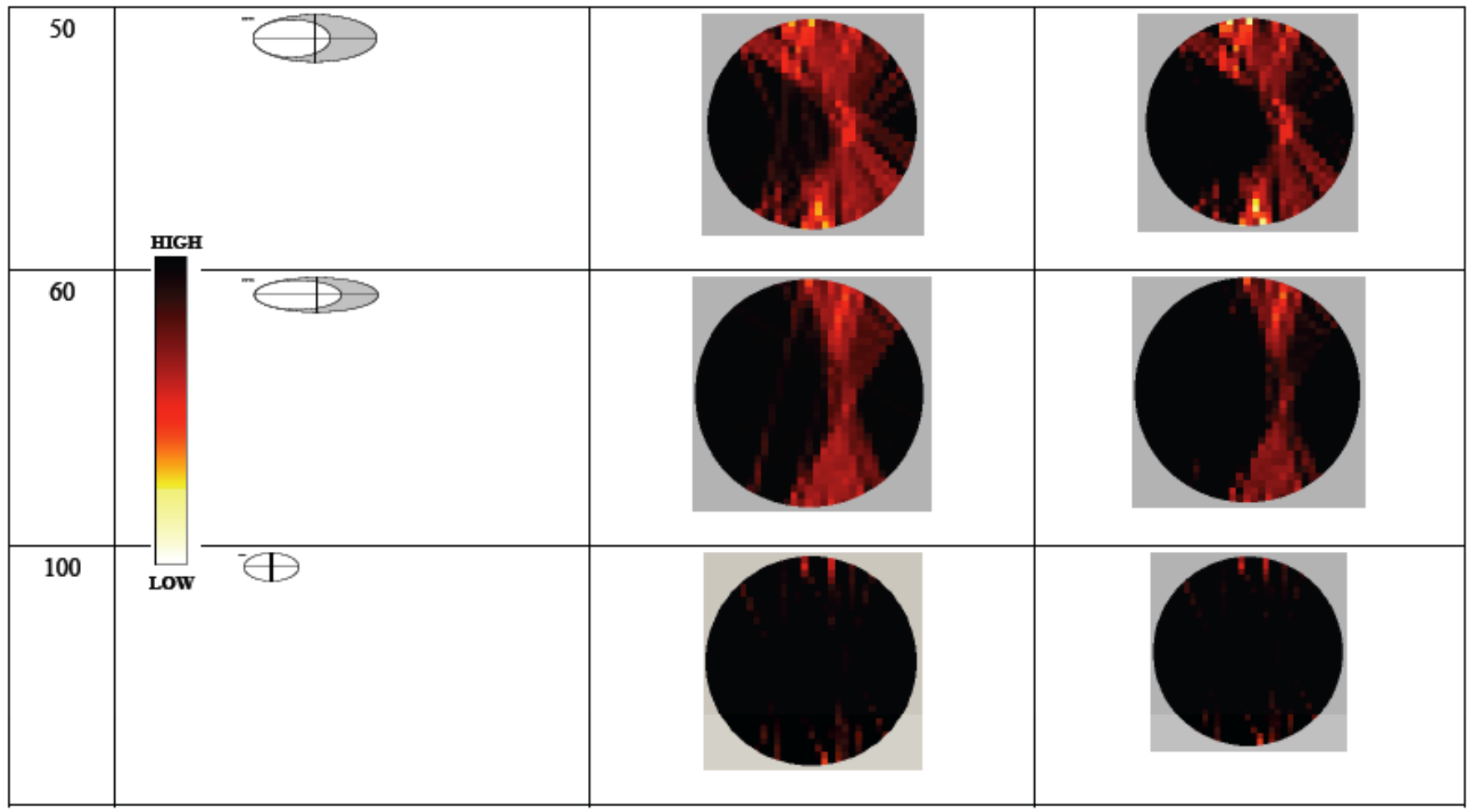

Fig. (13). Tomogram images for real-time plastic bead flow using filters. 


\section{DISCUSSIONS}

From the static and dynamic online experiments, it is seen that the designed optical fibre sensors are able to perform in real-time situations. In the static flow experiments using several opaque objects, it is observed that there are small differences between the obtained image and the modelled image. The factors that might contribute to the errors are because of the imprecise location of objects in sensor's zone and unexpected influence to the sensors such as internal and also external noise.

Meanwhile, for the dynamic flow experiments, the ideal image obtained by the sensors should be the same as the shape of the filter whereby the concentration of the plastic beads should be concentrated at the transparent area of the filters which allows solids to flow through. However, due to the abrasion and bouncing of the plastic beads within themselves as they flow through the distance of $12 \mathrm{~cm}$ before reaching the sensor's area, the reconstructed images show that the plastic beads do not concentrate in the area as it should be according to the filters. The further the distance from the filters to the sensors is, the plastic beads will experience more abrasion and bouncing, thus producing a more scattered flow.

\section{REFERENCES}

[1] Mohd Fua'ad Rahmat. Instrumentation of Particle Conveying Using Electrical Charge Tomography. Sheffield Hallam University: Ph.D. Thesis; 1996.

[2] Kak, A.C., Malcolm Slaney Principles of Computerized Tomographic Imaging. IEEE Press. New York; 1999.

[3] Abdul Rahim R, Chan KS, Pang JF, Leong LC. Application of optical tomography in real time monitoring for solid particles. J Adv Powder Technol 2005; 16 (4).
[4] Xu H, Dehghani H, Pogue PW, Springett R, Paulsen KD, Dunn, JF. Near-infrared imaging in the small animal brain: optimization of fiber positions. J Biomed Optics 2003; 8 (1): 102-110.

[5] Ramli, N. Tomographic imaging instrumentation of pneumatic particle conveying using optical fibre sensors. Sheffield Hallam University: MPhil/PhD Transfer Report; 1998.

[6] Abdul Rahim R. A tomography imaging system for pneumatic conveyors using optical fibres. Sheffield Hallam University: Ph.D. Thesis; 1996.

[7] Ibrahim S. Measurement of gas bubbles in a vertical water column using optical tomography. Sheffield Hallam University: Ph.D. Thesis; 2000.

[8] San CK. Real time image reconstruction for fan beam optical tomography system. Universiti Teknologi Malaysia: M.Sc. Thesis; 2002.

[9] Gamio JC, Oritz-Aleman C. The $3^{\text {rd }}$ World Congress on Industrial Process Tomography; 2003; Banff, Canada.

[10] Yan H, Liu LJ, Xu H, Shao FQ. Image reconstruction in electrical capacitance tomography using multiple linear regression and regularization. Meas Sci Technol 2001; 12: 575-581.

[11] Chan KS, Abdul Rahim R. The $2^{\text {nd }}$ World Engineering Congress; July 2002; Malaysia

[12] Warsito W, Fan LS. Neural network based multi-criterion optimization image reconstruction technique for imaging two- and threephase flow systems using electrical capacitance tomography. Meas Sci Technol 2001; 12: 2198-2210.

[13] Wang G, Vannier MW. Computerized tomography - encyclopedia of electrical and electronics engineering. United States: John Wiley \& Sons; 1998.

[14] Hielscher AH, Bartel S. Use of penalty terms in gradient-based iterative reconstruction schemes for optical tomography. J Biomed Optics 2001; 6 (2): 183-192.

[15] Salked, J, Yang WQ, Pendleton J. An iterative method for improving ECT images. Application Note AN4. U.K.: Process Tomography Ltd.; 1999.

[16] Abdul Rahim R, Leong LC, Chan KS, Pang JF. Optical tomography system using switch mode fan beam projection: modelling techniques. J Optical Eng 2005; 44 (12). 